\title{
Pulmonary actinomycosis complicating infliximab therapy for Crohn's disease
}

\author{
R D Cohen, W R Bowie, R Enns, J Flint, J M Fitzgerald
}

Thorax 2007;62:1013-1014. doi: 10.1136/thx.2006.075150

The use of anti-tumour necrosis factor (TNF) agents has expanded significantly over the past few years, particularly for rheumatological diseases and Crohn's disease. A number of associated opportunistic infections have been observed as a result of suppression of $T$ cell-mediated immunity, the most frequent being tuberculosis. We report the first case of pulmonary actinomycosis in a patient receiving regular infusions of infliximab, an anti-TNF agent, for Crohn's disease.

A 52-year-old man presented with a 12-day history of fever, night sweats and a non-productive cough. He had a 9-year history of stenosing Crohn's disease (CD) affecting the terminal ileum and had undergone four ileocolic resections. Infliximab $500 \mathrm{mg}$ every 9 weeks was started prophylactically following his last resection 4 years before the current illness, and his CD had remained well controlled. Other immunosuppressive medication included budesonide $6 \mathrm{mg}$ and 6-mercaptopurine $125 \mathrm{mg}$, both daily. There was a history of significant exposure to tuberculosis, although his Mantoux test was negative and the chest radiograph was normal before commencement of infliximab.

On examination his temperature was $38^{\circ} \mathrm{C}$. There were crackles over the left anterior thorax. A chest radiograph showed homogeneous segmental left upper lobe consolidation with no cavitation or pleural effusion. His haemoglobin was $149 \mathrm{~g} / \mathrm{l}$ with a white blood cell (WBC) count of $9.3 \times 10^{9} / 1$. Two sets of blood cultures were negative.

Fibreoptic bronchoscopy revealed an exophytic whitish mass in the left upper lobe bronchus. Cytological examination of the bronchial lavage fluid showed acute and chronic inflammatory cells and reactive pneumocytes. A Zeihl-Neelsen stain was negative, as were a methenamine silver stain for Pneumocystis and stains for fungi. A bronchial biopsy specimen showed a matted structure consistent with actinomycosis (fig 1).

Shortly after the bronchoscopy the patient developed a high fever with rigors and his oxygen saturation deteriorated. A CT scan of the chest revealed diffuse patchy consolidation, groundglass opacities and branching centrilobular nodular opacities, most severe in the left upper lobe; no cavitation was present (fig 2). He was admitted to hospital and started on penicillin $G$, 3 million units 4-hourly, clarithromycin $1 \mathrm{~g} /$ day orally and supplemental oxygen therapy. Twenty-four hours after admission the WBC rose to $15.2 \times 10^{9} / 1$ with 13.0 polymorphonuclear leucocytes. The fever and oxygenation improved over the next 4 days. The patient was subsequently discharged on penicillin $\mathrm{G}, 18$ million units daily by continuous intravenous infusion via a central venous catheter. He remained asymptomatic and a CT scan of the chest 3 weeks after discharge revealed almost complete resolution of the pulmonary infiltrates. The intravenous penicillin was discontinued after 5 weeks and oral doxycycline $100 \mathrm{mg}$ twice daily was commenced.
Acute and convalescent sera for Mycoplasma pneumoniae and Legionella pneumophila showed no rise in titre. Actinomyces graevenitzii was cultured from the bronchial lavage fluid; cultures for tuberculosis, fungi and viruses were negative.

\section{DISCUSSION}

Pulmonary actinomycosis is a relatively rare disease usually caused by Actinomyces israelii, but other pathogenic species have been reported. ${ }^{1}$ It usually presents as an indolent pneumonia with fever, weight loss, cough, sputum and chest pain. There are no specific radiographic manifestations but lesions that involve the chest wall and pleura with destruction of adjacent bones are highly suggestive. ${ }^{1}$ The symptoms and clinical and radiological signs often mimic malignancy or tuberculosis, and miliary presentations of the disease have been reported. ${ }^{2}$ Ariel et $a l^{3}$ described five patients with endobronchial actinomycosis presenting in a subacute fashion. Endobronchial infection is thought to be due to implantation of infected aspirated material or lympho-haematogenous spread to the peribronchial region.

Actinomycosis has occasionally been reported in association with immunosuppressed states including post solid organ transplantation, with HIV/AIDS and following chemotherapy, ${ }^{4-6}$ but most often no defects in immunity can be identified in patients with this infection. It remains unclear which host defence mechanisms are important in preventing or controlling actinomycosis and the degree to which the incidence of infection is increased in immunocompromised patients, if at all. $^{7}$ Dental procedures and aspiration of oropharyngeal contents are recognised risk factors for pulmonary actinomycosis, but these were absent in our patient. Actinomycosis has also occasionally been reported to complicate the diagnosis of $\mathrm{CD}$ of the small bowel; however, our patient had had multiple resections with no evidence of opportunistic infections in his resected specimens. There have not been any reports to date of actinomycosis complicating treatment with infliximab or other TNF inhibitors.

Infliximab is a chimeric $\operatorname{IgG}_{1}$ monoclonal anti-TNF antibody. It was the first anti-TNF agent to be approved by the FDA for induction and maintenance therapy in patients with moderate to severe CD refractory to conventional treatments. ${ }^{8}$ It has multiple suppressive effects on the immune response. After a single infusion in patients with steroid refractory ileocolonic CD, almost no neutrophils could be detected in colonic biopsy specimens and there was also a decrease in mononuclear cells. ${ }^{9}$ Aberrant colonic epithelial HLA-DR expression completely disappeared, and the percentage of intercellular adhesion molecule 1, lymphocyte function-associated antigen l-expressing and interleukin (IL)-4 and TNF positive lamina propria mononuclear cells sharply decreased. ${ }^{9}$ Infliximab also decreases the proinflammatory cytokines IL- 1 and IL- 6 and adhesion molecules E-selectin and intercellular adhesion molecule- $1 .{ }^{10}$

Abbreviations: $C D$, Crohn's disease; IL, interleukin; TNF, tumour necrosis factor; WBC, white blood cells 


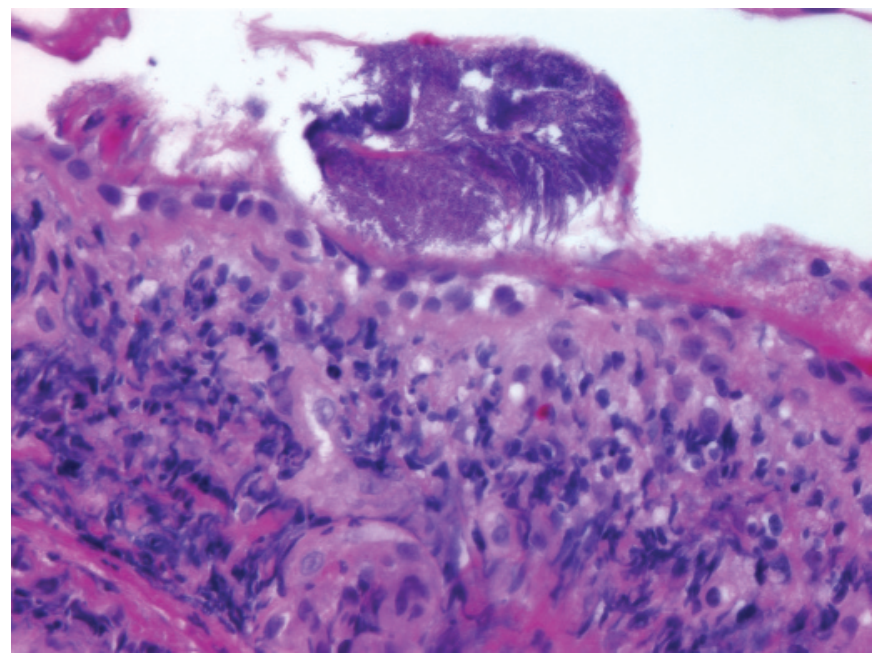

Figure 1 Endobronchial biopsy specimen showing actinomycotic mass with hyphae.

Infliximab has also received FDA approval for the treatment of rheumatoid arthritis, ankylosing spondylitis and psoriatic arthritis, and recent trials have demonstrated significant efficacy in the treatment of psoriasis and ulcerative colitis. ${ }^{11}{ }^{12}$ Infections associated with infliximab treatment have included tuberculosis (Mycobacterium tuberculosis and $M$ avium), leprosy, cryptococcosis, coccidioidomycosis, histoplasmosis, aspergillosis, candidiasis, Pneumocystis carinii (jiroveci) pneumonia, listeriosis and legionellosis. ${ }^{13}$ Although our patient presented with pulmonary symptoms, other patients receving anti-TNF agents may present with extrapulmonary manifestations. ${ }^{13}$

In summary, we have described the first case of pulmonary actinomycosis in association with infliximab therapy for CD. The patient presented acutely, rather than in the chronic manner typical of the disease in the lungs of normal nonimmunosuppressed individuals. This atypical presentation may have been due in part to the suppressive effects of infliximab on his normal inflammatory response. His response to high-dose intravenous penicillin was excellent and infliximab was restarted after a 4-month hiatus without consequence. Infliximab and other TNF inhibitors have shown promising therapeutic effects in an increasing number of conditions. Physicians caring for these individuals need to be aware of the entire spectrum of associated infectious complications and, in particular, need to maintain a high index of suspicion because of their often systemic and atypical presentation.

\section{Authors' affiliations}

R D Cohen, W R Bowie, R Enns, J Flint, J M Fitzgerald, Departments of Medicine and Pathology, University of British Columbia, Vancouver, Canada

Competing interests: None.

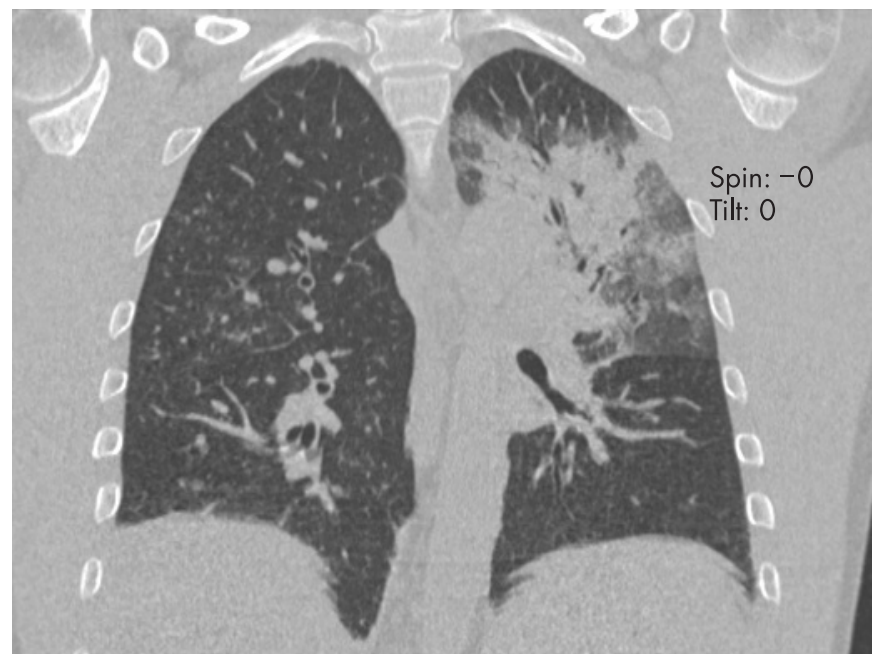

Figure 2 CT scan showing left upper lobe consolidation without cavitation.

Correspondence to: Dr Richard Cohen, Room 11223, Gordon \& Leslie Diamond Health Care Centre, 11 th Floor, 2775 Laurel Street, Vancouver, BC V5Z 1M9, Canada; richardcohen@shaw.ca

Received 20 December 2006

Accepted 28 March 2007

\section{REFERENCES}

1 Yildiz O, Doganay M. Actinomycoses and Nocardia pulmonary infections. Curr Opin Pulm Med 2006;12:228-34.

2 Fisher M. "Miliary" actinomycosis. J Can Assoc Radiologists 1980;31:149-50.

3 Ariel A, Brever R, Kamal N, et al. Endobronchial actinomycosis simulating bronchogenic carcinoma. Chest 1991;99:493-5.

4 Bassiri A, Girgis R, Theodore J. Actinomyces odontolyticus thoracopulmonary infections: two cases in lung and heart-lung transplant recipients and a review of the literature. Chest 1996; 109:1109-11.

5 Cendan I, Klapholz A, Talawera W. Pulmonary actinomycosis: a cause of endobronchial disease in a patient with AIDS. Chest 1993;103:1886-7.

6 Charalabopoulos K, Charalabopoulos AK, Papalimneou V, et al. Lung, pleural and colon actinomycosis in an immunocompromised patient: a rare form of presentation. Chemotherapy 2003;49:209-11.

7 Russo T. Agents of actinomycosis. In: Mandell G, Bennett J, Dolin R, eds. Mandell, Douglas and Bennett's principles and practice of infectious diseases, 6th edn. New York: Elsevier/Churchill Livingstone, 2005:2924-31.

8 Blonski W, Lichtenstein GR. Complications of biological therapy for inflammatory bowel diseases. Curr Opin Gastroenterol 2006;22:30-43.

9 Baert $F$, D'Haens $G$, Peeters $M$, et al. Tumor necrosis factor alpha antibody (infliximab) therapy profoundly down-regulates the inflammation in Crohn's ileocolitis. Gastroenterology 1999;116:22-8.

10 Bell S, Kamm M. The clinical role of anti-TNF alpha antibody treatment in Crohn's disease. Alimentary Pharmacol Ther 2000;14:501-14.

11 Reich K, Nestle F, Papp K, et al. Infliximab induction and maintenance therapy for moderate-to-severe psoriasis: a phase III, multicentre, double-blind trial. Lancet 2005;366:1367-74.

12 Sandborn W, Rachmilewitz D, Hanaver S, et al. Infliximab induction and maintenance therapy for ulcerative colitis: the ACT 2 trial. Gastroenterology 2005; 128:A-104

13 Crum NF, Lederman ER, Wallace MR. Infections associated with tumor necrosis factor-alpha antagonists. Medicine (Baltimore) 2005;84:291-302. 